\title{
The beneficial impacts of splanchnic vasoactive agents on hepatic functional recovery in massive hepatectomy porcine model
}

\author{
Hye-Sung Jo ${ }^{1 \#}$, Jae Hyun Han ${ }^{2 \#}$, Yoon Young Choi ${ }^{3}$, Jin-I Seok ${ }^{3}$, Young-In Yoon ${ }^{4}$, Dong-Sik Kim ${ }^{1}$ \\ ${ }^{1}$ Division of HBP Surgery and Liver Transplantation, Department of Surgery, Korea University College of Medicine, Seoul, Republic of Korea; \\ ${ }^{2}$ Department of Surgery, St. Vincent's Hospital, College of Medicine, The Catholic University of Korea, Seoul, Republic of Korea; ${ }^{3}$ Department of \\ Biomedical Science, Korea University College of Medicine Graduate School, Seoul, Republic of Korea; ${ }^{4}$ Division of Hepatobiliary Surgery and Liver \\ Transplantation, Department of Surgery, Asan Medical Center, University of Ulsan College of Medicine, Seoul, Republic of Korea \\ Contributions: (I) Conception and design: HS Jo, JH Han, DS Kim; (II) Administrative support: YY Choi, JI Seok; (III) Provision of study materials \\ or patients: JH Han, YI Yoon; (IV) Collection and assembly of data: HS Jo, YY Choi, JI Seok; (V) Data analysis and interpretation: HS Jo, JH Han, \\ YI Yoon, DS Kim; (VI) Manuscript writing: All authors; (VII) Final approval of manuscript: All authors. \\ "These authors contributed equally to this work. \\ Correspondence to: Prof. Dong-Sik Kim, MD, PhD. Division of HBP Surgery and Liver Transplantation, Department of Surgery, Korea University \\ College of Medicine, 73, Goryeodae-ro, Seongbuk-gu, Seoul 02841, Republic of Korea. Email: kimds1@korea.ac.kr.
}

Background: Excessive portal pressure after massive hepatectomy can cause hepatic sinusoidal injury and have deleterious impacts on hepatic functional recovery, contributing to developing post-hepatectomy liver failure. This study aimed to assess the effects of splanchnic vasoactive agents on hepatic functional recovery and regeneration while clarifying the underlying mechanism, using a 70\% hepatectomy porcine model.

Methods: Eighteen pigs undergoing 70\% hepatectomy were involved in this study and divided into three groups: control $(n=6)$, terlipressin $(n=6)$, and octreotide $(n=6)$. Terlipressin $(0.5 \mathrm{mg})$ and octreotide $(0.2 \mathrm{mg})$ were administered 3 times a day for each group with the first dose starting just before surgery until the 7th postoperative day, at which time the surviving pigs were sacrificed. During the period, portal pressure, liver weight, biochemical analysis, histological injury score, and molecular markers were evaluated and compared between groups.

Results: The 7-day survival rates in the octreotide, terlipressin, and control groups were $100 \%, 83.3 \%$, and $66.7 \%$, respectively. The portal pressures decreased in both terlipressin and octreotide groups than the control group at 30 minutes, 1 hour and 6 hours after hepatectomy. The amount of regeneration measured by liver weight to body weight ratio at the time of sacrifice in the terlipressin group was smaller than that in the control group (117\% vs. 129\%, P=0.03). Serum aspartate aminotransferase (AST) and total bilirubin levels at 1 and 6 hours after hepatectomy and prothrombin time/international normalized ratio (PT/INR) at 6 hours after hepatectomy were significantly improved in the terlipressin and octreotide groups compared to the control group. Serum endothelin-1 (ET-1) was significantly lower in the terlipressin group than that in the control group 6 hours after hepatectomy $(\mathrm{P}<0.01)$. The histological injury score in the control group was significantly higher than that in the terlipressin group on the 7 th postoperative day $(\mathrm{P}<0.01)$.

Conclusions: Splanchnic vasoactive agents, such as terlipressin and octreotide, could effectively decrease portal pressure and attenuate liver injury after massive hepatectomy.

Keywords: Hepatectomy; portal pressure; terlipressin; somatostatin

Submitted Jul 30, 2019. Accepted for publication Nov 12, 2019.

doi: $10.21037 /$ hbsn.2019.11.31

View this article at: http://dx.doi.org/10.21037/hbsn.2019.11.31 


\section{Introduction}

Liver resection has provided a curative chance for patients with primary or metastatic hepatic malignancy. Although advances in surgical techniques and postoperative care over the past decades have yielded noteworthy improvements in postoperative outcomes (1), post-hepatectomy liver failure (PHLF) remains one of the most important issues to be solved. However, no effective preventive measures or treatments have been established (2). When PHLF develops, only supportive care can be provided (3).

Excessive portal pressure after massive hepatectomy can damage the sinusoidal endothelium with the release of cytokines, consequently impairing liver regeneration and recovery $(4,5)$. Thus, several attempts have been made to modulate portal flow, especially in patients undergoing living donor liver transplantation with a small graft. Portocaval shunt, splenic artery embolization/ligation, and splenectomy have been clinically used to improve the function of a small graft by decreasing portal inflow $(6,7)$. However, these are invasive and irreversible interventions with a risk for morbidity. Furthermore, it is difficult to adjust the optimal level of portal flow as liver grafts regenerate because these procedures have to be performed during surgery.

Meanwhile, splanchnic vasoactive agents, such as somatostatin or vasopressin and their analogs, can induce splanchnic vasoconstriction with minimal influence on systemic circulation (8). Splanchnic vasoconstriction subsequently decreases portal flow and pressure, which may reduce sinusoidal endothelial injury and optimize recovery of a small remnant liver. Decreased portal pressure and marked survival improvement were observed after massive hepatectomy with the administration of splanchnic vasoactive agents in a $90 \%$ hepatectomy rat model (9). Considering the limitation of a small animal study in hemodynamic measurement, and interspecies differences, large animal studies are needed to obtain more detailed information. Thus, this study aimed to assess the effects of splanchnic vasoactive agents on hepatic functional recovery and regeneration while clarifying the underlying mechanism using a $70 \%$ hepatectomy porcine model. We present the following article in accordance with the ARRIVE reporting checklist (available at https://hbsn.amegroups.com/article/ view/10.21037/hbsn.2019.11.31/rc).

\section{Methods}

\section{Study design}

This study was approved by the Korea University Institutional
Animal Care and Use Committee (KUIACUC-2015-120) and conducted in accordance with the "Animal Research: Reporting in Vivo Experiments" guidelines. Eighteen domestic female pigs weighing between 28 and $40 \mathrm{~kg}$ were used in this study. They were housed in a temperature- and humiditycontrolled room and cared for according to the national guidelines for ethical animal research.

In this study, terlipressin and octreotide were used as a vasopressin analog and a long-acting somatostatin analog, respectively. Overall, 18 pigs were divided into three groups: control $(n=6)$, terlipressin $(n=6)$, and octreotide $(\mathrm{n}=6)$. The schematic diagram of the study design is demonstrated in Figure 1. The experimental doses of terlipressin and octreotide were determined as equal to those used in humans for treatment of variceal bleeding or hepatorenal syndrome (10). However, we reduced the dose of terlipressin by half because side effects such as peripheral cyanosis or sustained tachycardia occurred in initial cases in the terlipressin group. As a consequence, $0.5 \mathrm{mg}$ terlipressin or $0.2 \mathrm{mg}$ octreotide was injected intramuscularly three times a day from just before hepatectomy to 7 days after surgery for each group. All pigs were cared for until 7 days after surgery, at which time the surviving pigs were sacrificed.

\section{Surgical procedure for $70 \%$ bepatectomy in the porcine model}

All pigs fasted for 8 hours before general anesthesia induction. After initial sedation via injection of ketamine $(20 \mathrm{mg} / \mathrm{kg})$ and xylazine $(1.5 \mathrm{mg} / \mathrm{kg})$ to the forequarter paraspinal muscle, endotracheal intubation was performed. During surgery, inhalational anesthesia was maintained at $60 \% \mathrm{FiO}_{2}$ with $1.5 \%$ halothane and vital signs, including heart rate and oxygen saturation, were monitored.

The surgical procedures were detailed as follows. The pigs were placed in the supine position, and an upper midline incision from the level of the xiphoid process to just above the umbilicus was then created. After hilar dissection, the main portal vein was isolated, and the liver was mobilized by dividing all ligamentous attachments around the liver. Subsequently, the left lateral lobe and right and left median lobes were resected, leaving the caudate and right lateral lobe to achieve $70 \%$ resection of the entire liver volume based on the segmental anatomy of porcine liver $(11,12)$. The Glisson's pedicles to each lobe to be resected were isolated and divided individually. Along with the demarcation line according to the ischemic color change, 


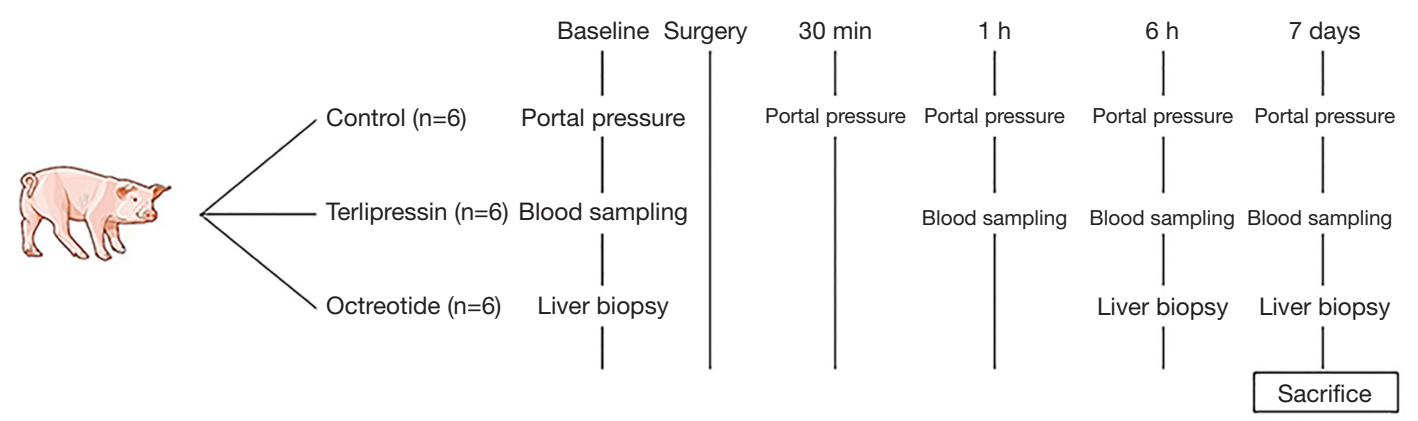

Figure 1 Schematic diagram of the study design. A total of 18 pigs were divided into three groups: control (n=6), terlipressin ( $\mathrm{n}=6$ ), and octreotide $(n=6)$. Portal pressure was measured, and blood sampling and liver biopsy were conducted according to the schedule shown.

the liver was transected using the clamp crushing technique, and the hepatic veins from each lobe were ligated and divided individually. After hemostasis was achieved, portal pressure was measured according to the predetermined schedule. Finally, abdominal closure was conducted.

\section{Hemodynamic measurements}

The portal pressure was measured before administration of terlipressin or octreotide and 30 minutes, and 1 and 6 hours after hepatectomy and on the 7th postoperative day before sacrifice. After dissection of the hepatic hilum, portal pressure was measured via direct puncture using an 18-gauge needle connected to an invasive pressure monitoring device (Vigileo Monitor, Edwards Lifesciences, Irvine, CA, USA).

\section{Biochemical analysis}

Blood samples were collected from the femoral vein before and 1 and 6 hours after hepatectomy and on the $7^{\text {th }}$ postoperative day. Serum aspartate aminotransferase (AST), alanine aminotransferase (ALT), total bilirubin levels and prothrombin time (PT) were measured using a biochemical analyzer (TBA-200FR NEO, Toshiba Medical Systems, Japan and Stago STAR Evolution, Stago, France).

\section{Enzyme-linked immunosorbent assay (ELISA)}

Serum levels of interleukin-6 (IL-6; Cusabio Biotech Company, USA), thymidine kinase (TK; Mybiosource, Inc., USA), endothelin-1 (ET-1; Cusabio Biotech Company), and endothelial nitric oxide synthase (eNOS; Enzo Life Sciences, USA) were directly determined using commercially available ELISA kits, according to the manufacturer's protocols. In brief, $50 \mu \mathrm{L}$ of 1:5 each diluted sample was placed in an antibody-coated well filled with $50 \mu \mathrm{L}$ of assay diluent and incubated for 1 hour at $37^{\circ} \mathrm{C}$. The wells were washed three times with PBS. The reaction was stopped by adding $50 \mu \mathrm{L}$ of stop solution. Optical density was determined at $450 \mathrm{~nm}$ using a microplate reader.

\section{Hepatic gene expression profiles in quantitative real-time reverse-transcription polymerase chain reaction $(q R T-P C R)$}

Expression of suppressor of cytokine signaling 3 (SOCS3) in liver tissues was evaluated using qRT-PCR. Liver tissues were stored at $-80^{\circ} \mathrm{C}$. RNAs were extracted from whole liver tissues using the total RNA Isolation Mini Kit (74104, Qiagen, Germany), then retranscribed into cDNA using the PrimeScript 1st strand cDNA Synthesis Kit (6110A, TAKARA, Japan). Real-time PCR were performed using the iCycler iQ system (Bio-Rad, USA) and TaqMan probe master mix by iQ Supermix (Bio-Rad, USA). Each sample was amplified in duplicate in 96-well plates using TaqMan probes [gene (assay ID number/NCBI reference sequence): SOCS3 (Ss03387992_u1/NM_001123196.1); GAPDH (Ss03374854_g1/NM_001206359.1); ThermoFisher, Waltham City, MA, USA]. Real-time PCR was performed under the following conditions: $1 \mathrm{~min}$ incubation at $60^{\circ} \mathrm{C}$, 10 min incubation at $95^{\circ} \mathrm{C}$ followed by 45 cycles composed of denaturation at $95^{\circ} \mathrm{C}$ for $15 \mathrm{~s}$ and annealing at $60^{\circ} \mathrm{C}$ for $1 \mathrm{~min}$. The relative quantification was performed, and the double delta $\mathrm{Ct}$ method was used for the calculation. All reactions were repeated four times in one sample. Data were normalized to the reference gene GAPDH. 


\begin{tabular}{|c|c|c|c|}
\hline & 9 & 1 & 2 \\
\hline Sinusoidal dilatation & $<5 \%$ & $5-50 \%$ & $>50 \%$ \\
\hline Cholestasis & No & Mild & Moderate \\
\hline Portal inflammation & No & Minimal & Mild \\
\hline Nuclear change & No & Present & \\
\hline Single cell necrosis & 0 in $5 \mathrm{HPF}$ & $1-10$ in 5 HPF & $>10$ in 5 HPF \\
\hline Confluent necrosis & No & Small in size and number & Large size and/or large number \\
\hline $\begin{array}{l}\text { Vacuolar degeneration } \\
\text { of cytoplasm of hepatocyte }\end{array}$ & No & $1-30 \%$ of all hepatocyte & $>30 \%$ of all hepatocyle \\
\hline
\end{tabular}

Figure 2 Histological injury score consisting of six different variables; each one has a score of 0-2 points assigned. HPF, high power field.

\section{Histological and immunobistochemical analyses}

Liver tissues were obtained before and 6 hours after hepatectomy and on the 7 th postoperative day. The fresh liver tissues were fixed in $10 \%$ formalin, and paraffinembedded sections $(4 \mu \mathrm{m})$ were stained with hematoxylineosin for histological assessment. The histological injury scoring system was developed based on previous studies $(13,14)$; it consisted of sinusoidal dilatation, cholestasis, portal inflammation, nuclear change, single cell necrosis, confluent necrosis, and vacuolar degeneration of hepatocyte cytoplasm (Figure 2). An experienced pathologist scored each variable from 0 to 2 points with blinding of group information. For immunochemistry, the paraffin-embedded tissue sections were dried overnight, deparaffinized, and rehydrated, followed by blocking of endogenous peroxidase with $3 \% \mathrm{H}_{2} \mathrm{O}_{2}$ solutions. Antigen was retrieved by heating the slides for 10 minutes. After diluted $(1: 1,000)$ anti-Ki-67 antibody (Ki-67/MKI67 Antibody, NB500170, Novus Biologicals, USA) was incubated overnight, biotinylated secondary antibody followed by avidin/biotinblocking solution was applied. The tissue sections were counterstained in hematoxylin (hematoxylin solution, Mayer's, MHS16, Sigma Aldrich, St. Louis, MO, USA). Finally, the number of Ki-67-positive cells on representative slides was assessed in four high-power fields for each slide.

\section{Statistical analysis}

All continuous variables were compared using linear mixed models for repeated measurement. The natural log transformation was used for variable that did not have a normal distribution. Kruskal-Wallis test followed by MannWhitney $\mathrm{U}$ test was performed in the comparison of Ki-67- positive cell number and histological injury score between groups. As the baseline portal pressure could be different depending on the hydration status and condition of each pig, the preoperative values of each group were corrected as a covariate in a linear mixed model to identify portal pressure change. Cumulative survival rates between groups were calculated using the Kaplan-Meier method. $\mathrm{P}$ values $<0.05$ were considered significant. All statistical analyses were performed using SAS 9.4 (SAS Institute, Cary, NC, USA).

\section{Results}

Two of 6 pigs in the control group and one of 6 pigs in the terlipressin group died before the scheduled time of sacrifice. The 7-day survival rates in the terlipressin and octreotide group did not differ significantly from that in the control group $(83.3 \%$ and $100 \%$ vs. $66.7 \%, \mathrm{P}=0.48$ and $\mathrm{P}=0.14$, respectively, Figure 3). An autopsy of pigs that died before the scheduled time of sacrifice did not reveal the specific cause of death.

\section{Portal pressure}

The portal pressures decreased in the terlipressin group than in the control group at 30 minutes, 1 and 6 hours after hepatectomy $(\mathrm{P}=0.03, \mathrm{P}=0.03$, and $\mathrm{P}=0.06)$; it also decreased in the octreotide group than in the control group at 30 minutes, 1 and 6 hours after hepatectomy $(\mathrm{P}=0.03$, $\mathrm{P}=0.03$, and $\mathrm{P}=0.04$ ) (Figure $4 A$ ). In the terlipressin and octreotide groups, mean portal pressure decreased until 6 hours after hepatectomy and rebounded on the 7 th postoperative day, as in the control group. No significant differences were observed between the terlipressin and 


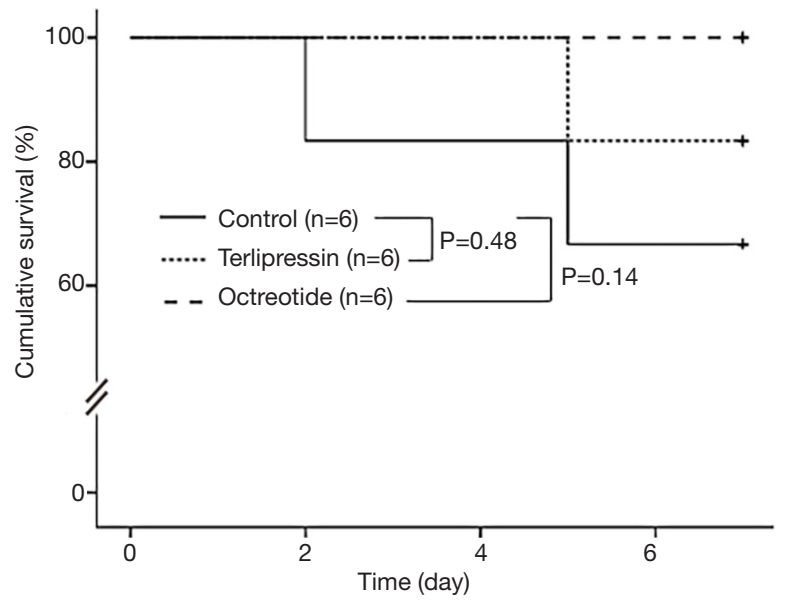

Figure 3 Cumulative survival curves in each group after $70 \%$ hepatectomy. The 7-day survival rates in the terlipressin and octreotide group did not differ significantly from that in the control group $(83.3 \%$ and $100 \%$ vs. $66.7 \%, \mathrm{P}=0.48$ and $\mathrm{P}=0.14$, respectively).

octreotide groups at each time point.

\section{Liver weight}

The resected liver weight to body weight ratio after hepatectomy did not differ significantly among the three groups $(\mathrm{P}=0.59)$, suggesting consistent resection of $70 \%$ of the total liver volume in each group (Figure $4 B$ ). The remnant liver weight immediately after resection was calculated from the weight of the resected liver, and liver weight at 1 week was measured directly after sacrifice. The increase in remnant liver weight to body weight ratio in the terlipressin group was less than that in the control group [0.75 to 1.63 (117\%) vs. 0.78 to 1.79 (129\%), $\mathrm{P}=0.03$ ].

\section{Biochemical analysis}

The results of the biochemical analysis according to the time points in each group are demonstrated in Figure 5. Serum AST level was lower in the terlipressin group than in the control group at 1 and 6 hours after hepatectomy $(\mathrm{P}=0.02$ and $\mathrm{P}=0.06$, respectively); it was also lower in the octreotide group than in the control group at the same time points, with borderline significance (both $\mathrm{P}=0.09$ ). Serum ALT level of all groups was within normal range at all time points, and no significant difference was observed both between groups and among time points. Total bilirubin

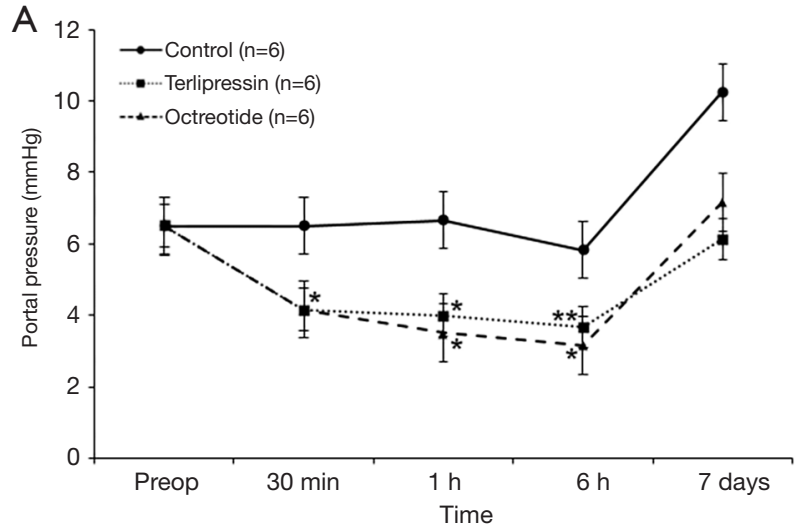

B

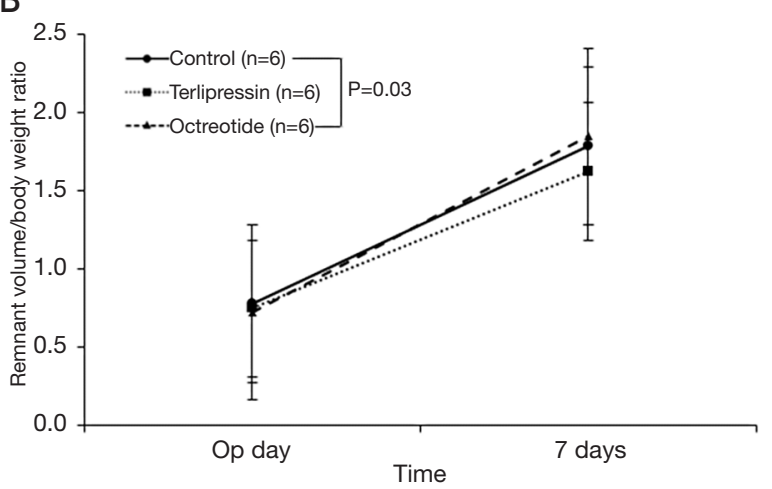

Figure 4 Postoperative change in portal pressure and remnant liver weight to body weight ratio. (A) Portal pressure in each group according to the predetermined schedule. Preoperative portal pressure was corrected; (B) remnant liver weight to body weight ratio in each group. Dots indicate the means, and whiskers indicate the standard error of the means. * $\mathrm{P}<0.05$ and **, $\mathrm{P}<0.1$ vs. control group in the same time point.

level was lower in the terlipressin group than in the control group at 1 and 6 hours after hepatectomy $(\mathrm{P}=0.09$ and 0.04 , respectively). Serum PT/international normalized ratio (PT/INR) was more prolonged in the control group than in the terlipressin and octreotide groups at 6 hours after hepatectomy with borderline significance (both $\mathrm{P}=0.07$ ).

\section{ELISA and qRT-PCR}

Serum ET-1 level was significantly lower in the terlipressin group than in the control group 6 hours after hepatectomy $(\mathrm{P}<0.01)$, while serum eNOS level did not differ significantly among groups at all time points. Regarding the liver regeneration signal, serum IL-6 level was significantly lower in the terlipressin group than in the octreotide group 

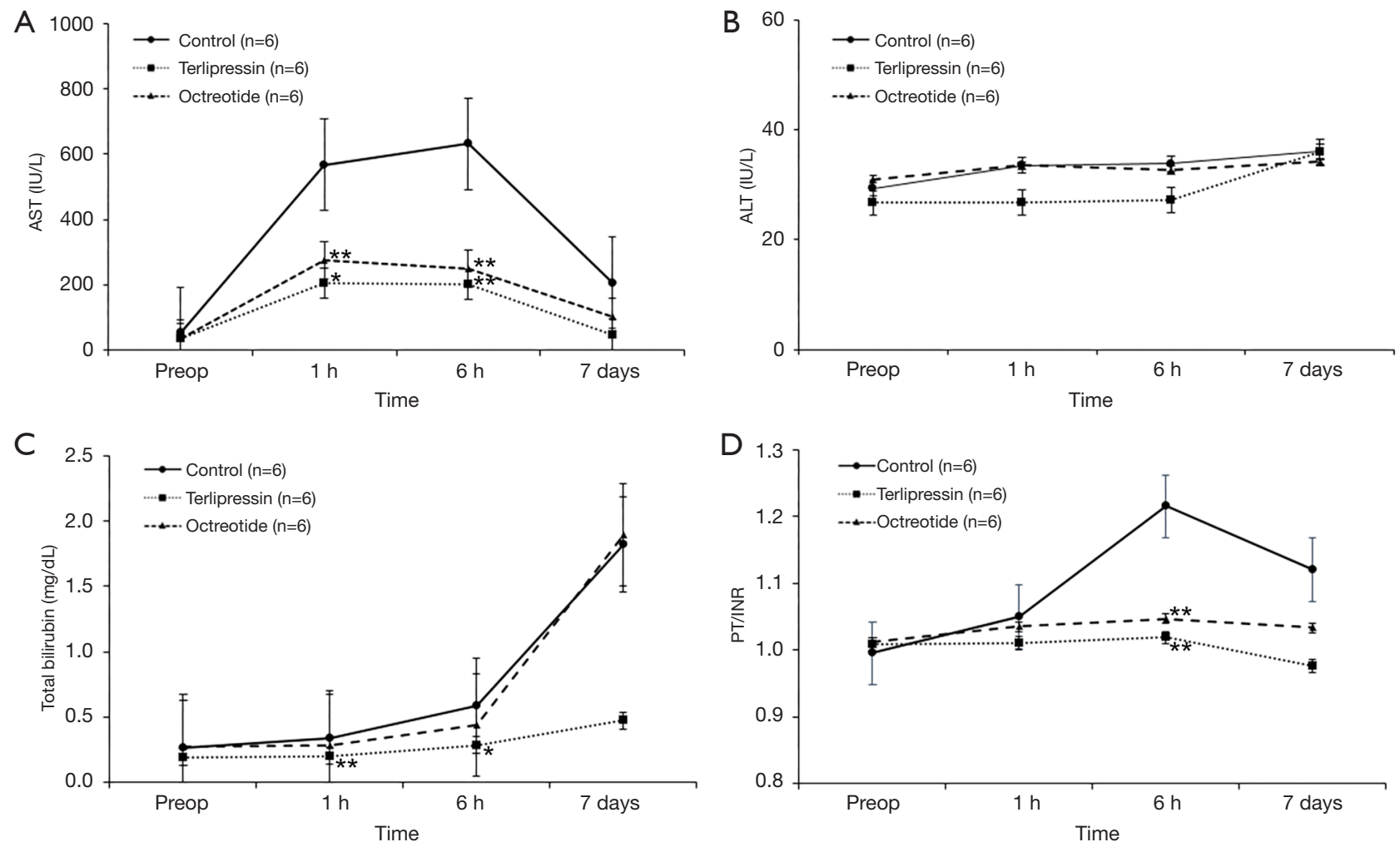

Figure 5 Postoperative change in serum aspartate aminotransferase (AST) (A), alanine aminotransferase (ALT) (B), total bilirubin levels (C), and prothrombin time/international normalized ratio (PT/INR) (D). Dots indicate the means, and whiskers indicate the standard error of the means. *, $\mathrm{P}<0.05$ and **, $\mathrm{P}<0.1$ vs. control group in the same time point.

on the 7 th postoperative day $(\mathrm{P}<0.01)$. Furthermore, serum level of TK, as a surrogate marker of liver regeneration, was significantly lower in the terlipressin group than in the control group on the 7 th postoperative day $(\mathrm{P}=0.046)$; it was higher in the octreotide group than in the control group 6 hours after hepatectomy, with borderline significance $(\mathrm{P}=0.09)$. The level of SOCS3 in liver tissues detected on qRT-PCR was lower in the terlipressin group than in the control group on the 7 th postoperative day, with borderline significance $(\mathrm{P}=0.07)$. Otherwise, no significant difference was observed among groups (Figure 6).

\section{Histological changes and Ki-67}

The representative hematoxylin-eosin-stained images in each group at the different time points are demonstrated in Figure $7 A$. The terlipressin and octreotide groups showed no significant areas of confluent necrosis, which was observed in the control group over time. The histological injury score in the control group was higher than those in the terlipressin and octreotide groups at 6 hours after hepatectomy with borderline significance (both $\mathrm{P}=0.07$ ), and on the 7 th postoperative day $(\mathrm{P}<0.01$ and $\mathrm{P}=0.07$, respectively) (Figure $7 B$ ). However, no significant difference was observed between the terlipressin and octreotide groups $(\mathrm{P}=0.12)$. Furthermore, the Ki-67-positive cell number in liver tissues was significantly higher in the control group than in the terlipressin and octreotide groups 6 hours after hepatectomy $(\mathrm{P}=0.02$ and $\mathrm{P}<0.01$, respectively) (Figure $7 C$ ).

\section{Discussion}

This experimental study identified the effects of splanchnic vasoactive agents on hepatic functional recovery and regeneration using a $70 \%$ hepatectomy porcine model. After massive hepatectomy, the small remnant liver strives to adapt to variable metabolic demands with its replicative capacity; otherwise, PHLF can occur and lead to serious complications (15). The small-for-size syndrome (SFSS), with a clinical presentation ranging from mild hepatic 

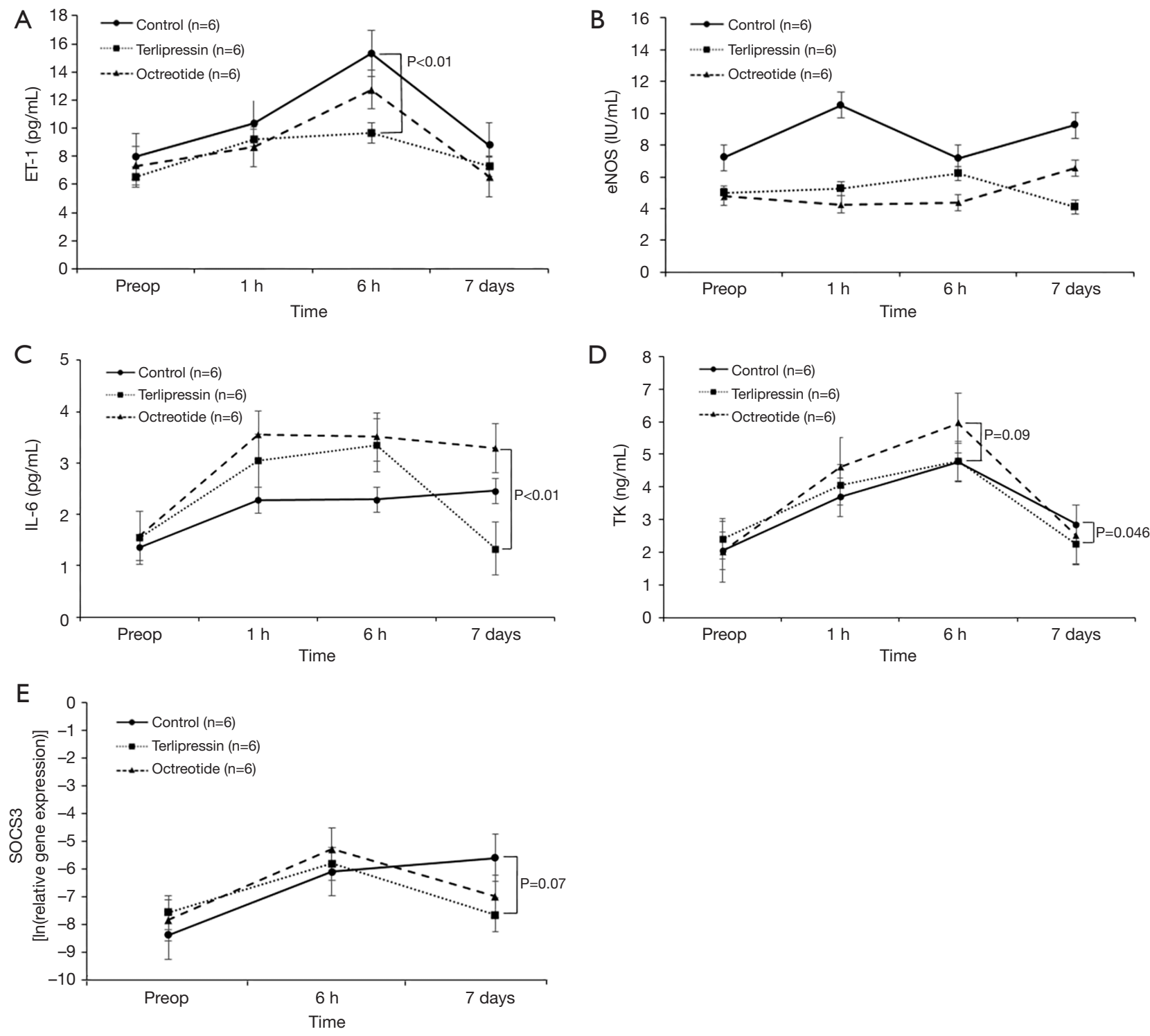

Figure 6 Postoperative change in levels of endothelin-1 (ET-1) (A), endothelial nitric oxide synthase (eNOS) (B), interleukin-6 (IL-6) (C), thymidine kinase (TK) (D) in serum, and suppressor of cytokine signaling 3 (SOCS3) in liver tissues (E). The natural log (ln) transformation was performed for SOCS3 to have a normal distribution. Dots indicate the least squares (LS) means, and whiskers indicate the standard error of the means.

dysfunction to irreversible graft failure in patients receiving living donor liver transplantation with a small-for-size graft, shares a certain pathophysiological process with PHLF as well (16-18). In both PHLF and SFSS, although shear stress in the hepatic sinusoids derived from the increase in portal flow acts as a stimulus for liver regeneration (19), excessive portal flow could cause barotrauma to the sinusoidal endothelium, leading to microcirculatory impairment, sinusoidal congestion, space of Disse destruction, and impaired regeneration (20).

There have been many attempts to modulate excessive portal flow. Hemi-portocaval shunt could reduce the risk of complications and graft dysfunction in a small-for-size graft $(7,21)$. The splenic artery embolization/ligation or even splenectomy has been proposed to relieve portal overperfusion and to improve prognosis (6). However, these invasive methods can cause potential complications, such as massive colliquation of the spleen and septic shock (22). In 

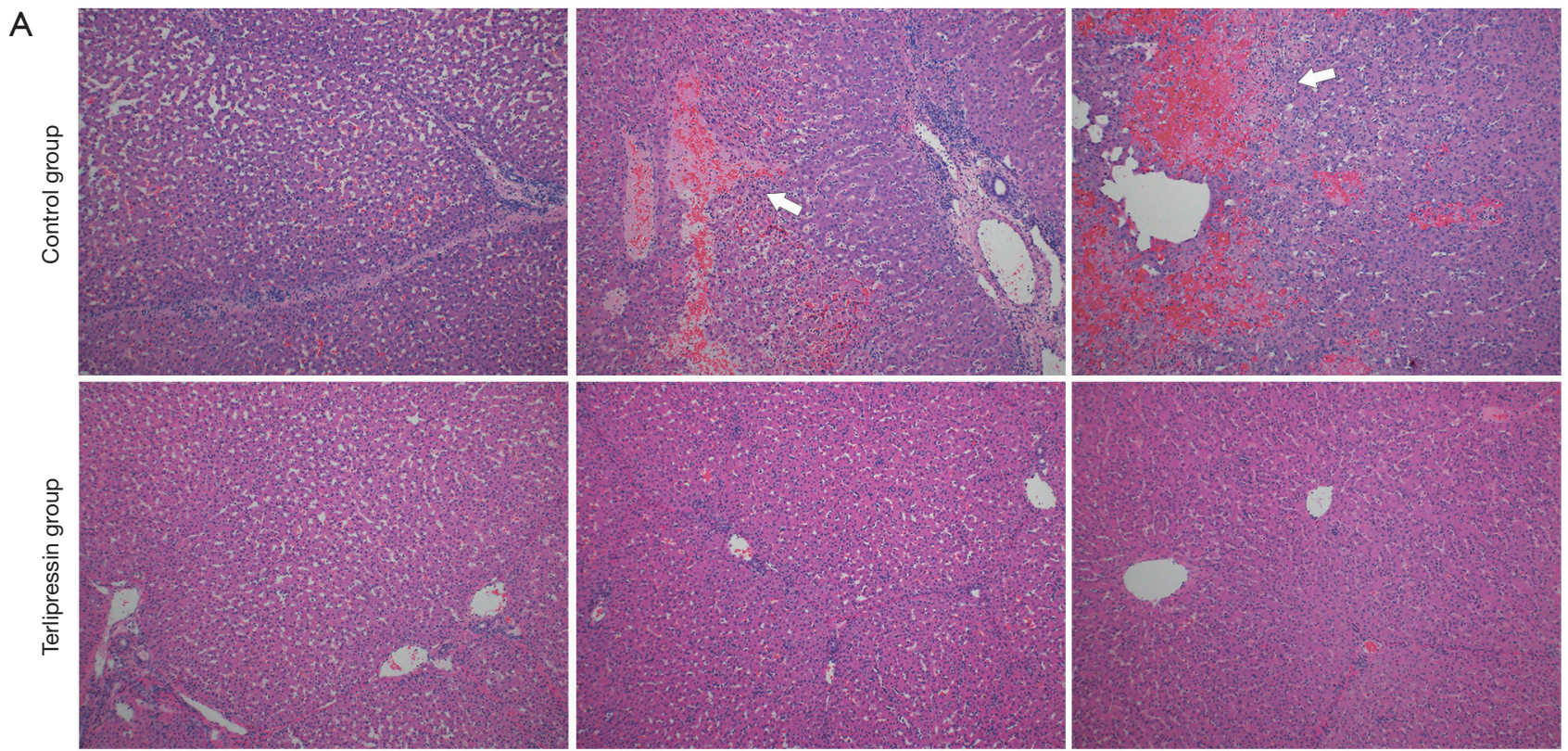

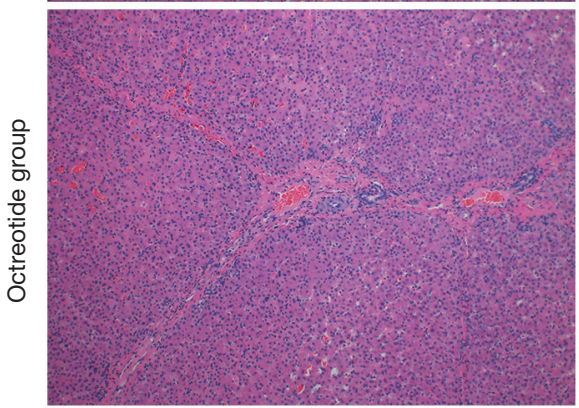

Preop

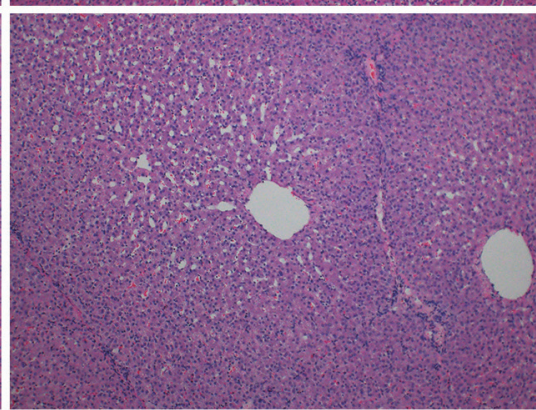

$6 \mathrm{~h}$

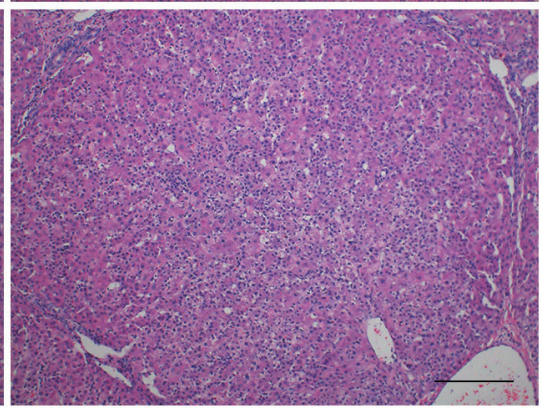

7 days
B

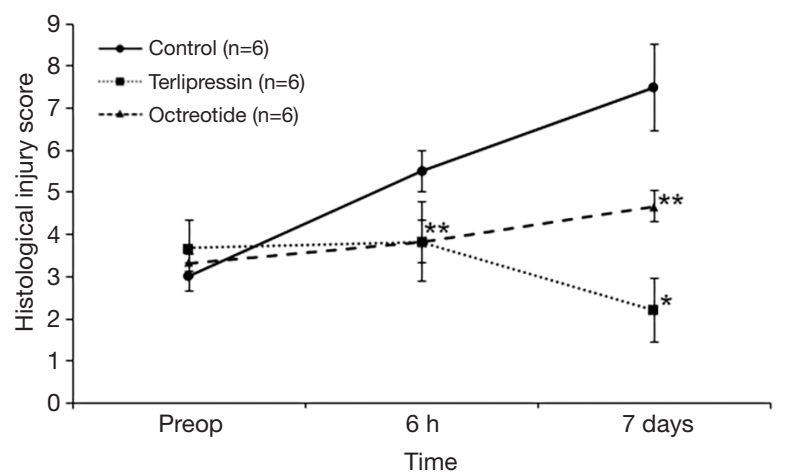

C

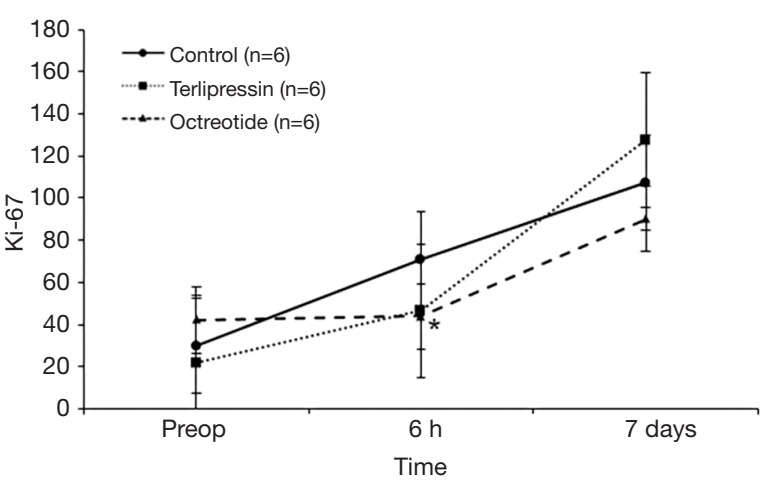

Figure 7 Histological and immunohistochemical analyses. (A) Representative hematoxylin-eosin staining in each group according to the predetermined schedule $(\times 100)$. White arrow indicates confluent necrosis and hemorrhage, which were observed in the control group but not in the terlipressin and octreotide groups. (B,C) Postoperative change in histological injury score (B) and Ki-67-positive cell number in the liver tissue $(\mathrm{C})$ in each group. * $\mathrm{P}<0.05$ and ${ }^{* *}, \mathrm{P}<0.1$ vs. control group in the same time point. 
present, various surgical modulations of portal flow are used to prevent SFSS after living donor liver transplantation and it has been suggested to be effective $(6,7,21)$. One study performed comparative study of the effects of terlipressin versus splenectomy after partial hepatectomy using a rat model (23). However, they failed to demonstrate the beneficial effects of portal modulation and has a limitation of using a small animal model. Although it is difficult to directly compare the effects of two methods, pharmacologic modulation could have particular advantages that it is noninvasive method without potential serious complications and could be adjusted to different clinical situations. When liver regeneration finishes and liver volume has been increased, the liver may suffer from relative portal insufficiency due to fixed portal flow from procedures which reduced portal flow mechanically.

In this study, terlipressin and octreotide were used as splanchnic vasoconstrictors. Terlipressin induces splanchnic arterial constriction by stimulating V1a vasopressin receptors expressed at the surface of vascular smooth muscle cells, subsequently decreasing portal pressure (24). It can be used more safely than vasopressin because it rarely causes serious systemic vascular complications and has been widely used in cirrhotic patients with variceal bleeding or hepatorenal syndrome (25). Although we reduced the dose of terlipressin because of its side effects, it is thought to be due to the lower weight of pigs and interspecies difference. Fahrner et al. reported that portal pressure increased with the extent of liver resection, and terlipressin reduced elevated portal pressure after partial hepatectomy and improved liver regeneration in their mouse model (26). Octreotide, a long-acting analog of somatostatin, also causes splanchnic vasoconstriction and decreases hepatic blood flow without affecting systemic circulation $(8,27)$. Hessheimer et al. identified that somatostatin not only offers reversible methods to reduce portal flow and protect the hepatic sinusoid but also induces cytoprotective effects, as observed in their small-for-size liver transplantation porcine model (28).

Portal pressure in the terlipressin and octreotide groups was significantly lower than in the control group, suggesting a potential role of splanchnic vasoactive agents in reducing excessive portal pressure. One unexpected result was that portal pressure, even in the control group, did not increase significantly until after 6 hours. However, several studies have demonstrated that portal pressure after $70 \%$ hepatectomy does not markedly increase in the immediate postoperative period $(29,30)$. The portal pressure had a tendency to increase on the 7 th postoperative day in all groups without significant differences. However, it is difficult to conclude clearly because the portal pressures could not be measured serially for 7 days owing to the limitation of a large animal study.

In this study, various results highly suggest attenuation of liver injury in the splanchnic vasoactive agent groups. Serum AST level, indicating hepatocyte injury, was significantly lower in the treatment groups. The absence of confluent necrosis and hemorrhage (which were observed in the control group) and lower histological injury score in the treatment groups support that splanchnic vasoactive agents could reduce liver injury after massive hepatectomy. Furthermore, the terlipressin group showed a lower ET-1 level than the control group. ET-1, a potent vasoconstrictive peptide, is upregulated in liver injuries and affects both stellate cell proliferation and smooth muscle $\alpha$-actin expression, which are important components during liver injury (31-33). Feng et al. reported that downregulation of ET-1 by somatostatin improves liver function in patients undergoing living donor liver transplantation (34). Meanwhile, the level of eNOS, an isoform of NO regulating sinusoidal blood flow and vascular tone, increased in response to increased shear stress and portal pressure after partial hepatectomy $(35,36)$. Although no significant differences were observed, the overall decreased eNOS levels in the treatment groups might indicate that vasoactive agents could effectively decrease immediate excessive portal pressure and liver injury.

Decreased portal inflow induced by splanchnic vasoactive agents modulates liver regeneration. IL-6 and TK levels, as molecular markers of liver regeneration, were lower in the terlipressin group than in the control group on the 7 th postoperative day. Furthermore, a decreased number of Ki-67-positive cells in liver tissues in the treatment groups consistently suggest that liver regeneration was relatively suppressed in the terlipressin group, compared with the control group. These consequently led to a smaller increase in remnant liver weight to body weight ratio in the terlipressin group. Previous studies have reported that portal flow modulation increase liver regeneration, and there have been attempts only to increase liver regeneration (26). However, whether increases volumetric regeneration necessarily leads to functional regeneration of the liver after massive hepatectomy remains controversial $(37,38)$. In this study, the terlipressin group showed lesser liver injury than the control group, despite a smaller increase in liver regeneration. 
The level of SOCS3, as negative regulator of liver regeneration, was lower in the terlipressin group than in the control group on the 7 th postoperative day. It could be inferred that negative feedback was activated on the 7 th postoperative day since excessive liver regeneration occurred in the control group while terlipressin group still continued to undergo well-controlled liver regeneration.

Different genes and cytokines were activated in the early phase after massive hepatectomy, and quiescent hepatocytes enter the cell cycle and DNA replication for regeneration; these hepatocytes are considered less functional owing to fewer sinusoids and bile canaliculi present until reformation of the normal liver microarchitecture $(28,39,40)$. The regeneration process is a unique feature of the liver, and it is mandatory for recovery of the small remnant liver after massive hepatectomy. However, if too many hepatocytes enter the cell cycle and lose their function before completing remodeling of the liver, severe liver failure can occur irreversibly. This might suggest that well-controlled liver regeneration in the early postoperative period could be better than excessive regeneration derived from the initial high portal flow.

In this study, the $70 \%$ hepatectomy model was used to identify the effects of vasoactive agents after massive hepatectomy. Although an extremely larger volume of the liver could be resected, the pigs could not survive for more than 2 days (41). Therefore, we employed a $70 \%$ hepatectomy model, considering that a remnant liver of at least $25 \%$ of the total liver volume is mandatory in the clinical setting, and the pigs had to survive for at least 1 week to assess the effect of splanchnic vasoactive agents on liver regeneration $(42,43)$.

Since these splanchnic vasoactive agents have been in clinical use widely for the treatment of complicated conditions with low profile side effects $(25,27)$, the beneficial effects shown in this study suggest potentials for use in prevention and treatment of PHLF or SFSS. Furthermore, if future study using more extensive hepatectomy models can demonstrate their beneficial effects on survival, it may expand limitation of current surgical indications enabling more extensive liver resections or partial graft liver transplantation with smaller volume.

One limitation of this study is that the time interval between 6 hours after hepatectomy and the 7 th postoperative day is relatively long, which makes the interpretation of results limited. It might be better if blood sampling and measurement of portal pressure were performed at shorter intervals until the time of sacrifice to understand the hemodynamic change and mechanism of effects of the splanchnic vasoactive agents. In addition, liver regeneration occurs as a consequence of complex interaction of many cytokines and molecules. Future studies are warranted for a detailed evaluation of molecular mechanisms which associated with liver regeneration in the setting of pharmacotherapy. Another limitation could be that in this study drug experimentation was not conducted at different doses.

In conclusion, splanchnic vasoactive agents could effectively decrease portal pressure and attenuate liver injury after massive hepatectomy. Considering their notable effects, further studies are warranted to assess the effect of splanchnic vasoactive agents using more extensive hepatectomy models.

\section{Acknowledgments}

Funding: This investigation was supported by the National Research Foundation of Korea (NRF) grant funded by the Korea government (MSIT; Ministry of Education, Science and Technology) (No. NRF-2017R1A2B2005754).

\section{Footnote}

Reporting Checklist: The authors have completed the ARRIVE reporting checklist. Available at https://hbsn. amegroups.com/article/view/10.21037/hbsn.2019.11.31/rc

Data Sharing Statement: Available at https://hbsn. amegroups.com/article/view/10.21037/hbsn.2019.11.31/dss

Conflicts of Interest: All authors have completed the ICMJE uniform disclosure form (available at https://hbsn.amegroups. com/article/view/10.21037/hbsn.2019.11.31/coif). Dr. DSK reports grants from National Research Foundation of Korea (NRF) funded by the Korea government (MSIT; Ministry of Education, Science and Technology) (No. NRF2017R1A2B2005754) during the conduct of the study. The other authors have no conflicts of interest to declare.

Ethical Statement: The authors are accountable for all aspects of the work in ensuring that questions related to the accuracy or integrity of any part of the work are appropriately investigated and resolved. This study was approved by the Korea University Institutional Animal Care and Use Committee (KUIACUC-2015-120) and conducted in accordance with the "Animal Research: Reporting in 
Vivo Experiments" guidelines.

Open Access Statement: This is an Open Access article distributed in accordance with the Creative Commons Attribution-NonCommercial-NoDerivs 4.0 International License (CC BY-NC-ND 4.0), which permits the noncommercial replication and distribution of the article with the strict proviso that no changes or edits are made and the original work is properly cited (including links to both the formal publication through the relevant DOI and the license). See: https://creativecommons.org/licenses/by-nc-nd/4.0/.

\section{References}

1. Jarnagin WR, Gonen $M$, Fong $Y$, et al. Improvement in perioperative outcome after hepatic resection: analysis of 1,803 consecutive cases over the past decade. Ann Surg 2002;236:397.

2. Fukushima K, Fukumoto T, Kuramitsu K, et al. Assessment of ISGLS definition of posthepatectomy liver failure and its effect on outcome in patients with hepatocellular carcinoma. J Gastrointest Surg 2014;18:729-36.

3. van den Broek MA, Olde Damink SW, Dejong CH, et al. Liver failure after partial hepatic resection: definition, pathophysiology, risk factors and treatment. Liver Int 2008;28:767-80.

4. Niiya T, Murakami M, Aoki T, et al. Immediate increase of portal pressure, reflecting sinusoidal shear stress, induced liver regeneration after partial hepatectomy. J Hepatobiliary Pancreat Surg 1999;6:275-80.

5. Sato Y, Koyama S, Tsukada K, et al. Acute portal hypertension reflecting shear stress as a trigger of liver regeneration following partial hepatectomy. Surg Today 1997;27:518-26.

6. Umeda Y, Yagi T, Sadamori H, et al. Effects of prophylactic splenic artery modulation on portal overperfusion and liver regeneration in small-for-size graft. Transplantation 2008;86:673-80.

7. Troisi R, Ricciardi S, Smeets P, et al. Effects of hemiportocaval shunts for inflow modulation on the outcome of small-for-size grafts in living donor liver transplantation. Am J Transplant 2005;5:1397-404.

8. Baik SK, Jeong PH, Ji SW, et al. Acute hemodynamic effects of octreotide and terlipressin in patients with cirrhosis: a randomized comparison. Am J Gastroenterol 2005;100:631-5.

9. Kim DS, Ji WB, Han JH, et al. Effects of splanchnic vasoconstrictors on liver regeneration and survival after
90\% rat hepatectomy. Ann Surg Treat Res 2018;94:118-28.

10. Silvain C, Carpentier S, Sautereau D, et al. Terlipressin plus transdermal nitroglycerin vs. octreotide in the control of acute bleeding from esophageal varices: a multicenter randomized trial. Hepatology 1993;18:61-5.

11. Court FG, Wemyss-Holden SA, Morrison CP, et al. Segmental nature of the porcine liver and its potential as a model for experimental partial hepatectomy. Br J Surg 2003;90:440-4.

12. Kahn D, Hickman R, Terblanche J, et al. Partial hepatectomy and liver regeneration in pigs--the response to different resection sizes. J Surg Res 1988;45:176-80.

13. Alexakis N, Gakiopoulou H, Dimitriou C, et al. Liver histology alterations during carbon dioxide pneumoperitoneum in a porcine model. Surg Endosc 2008;22:415-20.

14. Demetris AJ, Kelly DM, Eghtesad B, et al. Pathophysiologic observations and histopathologic recognition of the portal hyperperfusion or small-for-size syndrome. Am J Surg Pathol 2006;30:986-93.

15. Rahbari NN, Garden OJ, Padbury R, et al. Posthepatectomy liver failure: a definition and grading by the International Study Group of Liver Surgery (ISGLS). Surgery 2011;149:713-24.

16. Dahm F, Georgiev P, Clavien PA. Small-for-size syndrome after partial liver transplantation: definition, mechanisms of disease and clinical implications. Am J Transplant 2005;5:2605-10.

17. Golriz M, Majlesara A, El Sakka S, et al. Small for Size and Flow (SFSF) syndrome: An alternative description for posthepatectomy liver failure. Clin Res Hepatol Gastroenterol 2016;40:267-75.

18. Serenari M, Cescon M, Cucchetti A, et al. Liver function impairment in liver transplantation and after extended hepatectomy. World J Gastroenterol 2013;19:7922-9.

19. Sato Y, Tsukada K, Hatakeyama K. Role of shear stress and immune responses in liver regeneration after a partial hepatectomy. Surg Today 1999;29:1-9.

20. Man K, Fan ST, Lo CM, et al. Graft injury in relation to graft size in right lobe live donor liver transplantation: a study of hepatic sinusoidal injury in correlation with portal hemodynamics and intragraft gene expression. Ann Surg 2003;237:256-64.

21. Botha JF, Langnas AN, Campos BD, et al. Left lobe adultto-adult living donor liver transplantation: small grafts and hemiportocaval shunts in the prevention of small-for-size syndrome. Liver Transpl 2010;16:649-57.

22. Gonzalez HD, Liu ZW, Cashman S, et al. Small for 
size syndrome following living donor and split liver transplantation. World J Gastrointest Surg 2010;2:389.

23. Ulmer TF, Weiland A, Lurje G, et al. Comparative study of the effects of terlipressin versus splenectomy on liver regeneration after partial hepatectomy in rats. Hepatobiliary Pancreat Dis Int 2017;16:506-11.

24. Moreau R, Lebrec D. The use of vasoconstrictors in patients with cirrhosis: type $1 \mathrm{HRS}$ and beyond. Hepatology 2006;43:385-94.

25. Krag A, Borup T, Møller S, et al. Efficacy and safety of terlipressin in cirrhotic patients with variceal bleeding or hepatorenal syndrome. Adv Ther 2008;25:1105-40.

26. Fahrner R, Patsenker E, De Gottardi A, et al. Elevated liver regeneration in response to pharmacological reduction of elevated portal venous pressure by terlipressin after partial hepatectomy. Transplantation 2014;97:892-900.

27. Eriksson LS, Brundin T, Söderlund C, et al. Haemodynamic effects of a long-acting somatostatin analogue in patients with liver cirrhosis. Scand J Gastroenterol 1987;22:919-25.

28. Hessheimer AJ, Escobar B, Muñoz J, et al. Somatostatin therapy protects porcine livers in small-for-size liver transplantation. Am J Transplant 2014;14:1806-16.

29. Golriz M, El Sakka S, Majlesara A, et al. Hepatic hemodynamic changes following stepwise liver resection. J Gastrointest Surg 2016;20:587-94.

30. Bucur P, Bekheit M, Audebert C, et al. Simplified technique for $75 \%$ and $90 \%$ hepatic resection with hemodynamic monitoring in a large white swine model. J Surg Res 2017;209:122-30.

31. Gandhi CR, Harvey SA, Olson MS. Hepatic effects of endothelin: metabolism of [125I] endothelin-1 by liverderived cells. Arch Biochem Biophys 1993;305:38-46.

32. Rockey DC, Fouassier L, Chung JJ, et al. Cellular localization of endothelin-1 and increased production in

Cite this article as: Jo HS, Han JH, Choi YY, Seok JI, Yoon YI, Kim DS. The beneficial impacts of splanchnic vasoactive agents on hepatic functional recovery in massive hepatectomy porcine model. HepatoBiliary Surg Nutr 2021;10(3):325-336. doi: $10.21037 /$ hbsn.2019.11.31 liver injury in the rat: potential for autocrine and paracrine effects on stellate cells. Hepatology 1998;27:472-80.

33. Kuddus RH, Nalesnik MA, Subbotin VM, et al. Enhanced synthesis and reduced metabolism of endothelin-1 (ET1) by hepatocytes-an important mechanism of increased endogenous levels of ET-1 in liver cirrhosis. J Hepatol 2000;33:725-32.

34. Feng ZY, Xu X, Wu LJ, et al. Downregulation of endothelin-1 by somatostatin improves liver function of recipients undergoing adult-to-adult living donor liver transplantation. Chin Med J (Engl) 2010;123:1961-6.

35. Rockey DC, Shah V. Nitric oxide biology and the liver: report of an AASLD research workshop. Hepatology 2004;39:250-7.

36. Schoen JM, Wang HH, Minuk GY, et al. Shear stressinduced nitric oxide release triggers the liver regeneration cascade. Nitric Oxide 2001;5:453-64.

37. Ninomiya M, Shirabe K, Terashi T, et al. Deceleration of regenerative response improves the outcome of rat with massive hepatectomy. Am J Transplant 2010;10:1580-7.

38. Golse N, Bucur PO, Adam R, et al. New paradigms in post-hepatectomy liver failure. J Gastrointest Surg 2013;17:593-605.

39. Wack KE, Ross MA, Zegarra V, et al. Sinusoidal ultrastructure evaluated during the revascularization of regenerating rat liver. Hepatology 2001;33:363-78.

40. Fausto N. Liver regeneration. J Hepatol 2000;32:19-31.

41. Gaub J, Iversen J. Rat liver regeneration after $90 \%$ partial hepatectomy. Hepatology 1984;4:902-4.

42. Ferrero A, Viganò L, Polastri R, et al. Postoperative liver dysfunction and future remnant liver: where is the limit? World J Surg 2007;31:1643-51.

43. Golriz M, Ashrafi M, Khajeh E, et al. Establishing a Porcine Model of Small for Size Syndrome following Liver Resection. Can J Gastroenterol Hepatol 2017;2017:5127178. 